\title{
Paediatric chronic suppurative otitis media in the Free State Province: Clinical and audiological features
}

\author{
N J Tiedt, ${ }^{1}$ FCORL (SA), MMed (ORL); I R T Butler, ${ }^{1}$ MMed (ORL); U M Hallbauer, ${ }^{2}$ MPraxMed, DCH, MMed (Paed), FCPaed (SA); \\ M D Atkins, ${ }^{2}$ MMed (Paed); E Elliott, ${ }^{3}$ DTH, Dip HIV Mx, FCPath (Micro) (SA), MMed (Micro); M Pieters, ${ }^{3}$ MMed (Micro); \\ G Joubert, ${ }^{4}$ BA, MSc; R Y Seedat, ${ }^{1}$ MMed (ORL), FCORL (SA) \\ ${ }^{1}$ Department of Otorhinolaryngology, Faculty of Health Sciences, University of the Free State, Bloemfontein, South Africa \\ ${ }^{2}$ Department of Paediatrics and Child Health, Faculty of Health Sciences, University of the Free State, Bloemfontein, South Africa \\ ${ }^{3}$ Department of Medical Microbiology, Faculty of Health Sciences, University of the Free State, Bloemfontein, South Africa \\ ${ }^{4}$ Department of Biostatistics, Faculty of Health Sciences, University of the Free State, Bloemfontein, South Africa
}

Corresponding author: R Y Seedat (seedatry@ufs.ac.za)

Background. Chronic suppurative otitis media (CSOM) is a chronic infection of the middle ear cleft. In sub-Saharan Africa $>50 \%$ of cases occur in children $<10$ years of age.

Objectives. To describe the otological, audiological and bacteriological findings in children with CSOM.

Methods. We conducted a prospective study at the Ear, Nose and Throat (ENT) Clinic at Universitas Academic Hospital between August 2009 and December 2010. We included all children with CSOM over this period. Patients underwent ENT and paediatric examination, and were tested for HIV. Pus swabs were taken after an ear toilet for routine microbiology, fungal and Mycobacterium tuberculosis culture. We performed audiological testing after the otorrhoea had resolved.

Results. Eighty-six children (113 ears) were included, with a median age of 4.6 years (range 1 - 12 years). The mean duration of otorrhoea was 161.7 weeks (range 4 - 572 weeks). Nine patients (10.5\%) presented with coalescent mastoiditis and/or intracranial complications of CSOM. Of the 153 organisms identified, Gram-negative bacteria were present in 93 (82.3\%) ears, with $94.8 \%$ of these being sensitive to quinolones. Only 1 case of tuberculous otitis media was identified. HIV infection was present in $54.6 \%$ of patients tested. There was a hearing loss in 44 (66.7\%) of the tested affected ears.

Conclusions. There was a long delay between the onset of symptoms and accessing ENT services. Most cases of CSOM were due to quinolone-sensitive Gram-negative aerobes. There was a high prevalence of cholesteatoma, hearing loss and other complications in children in this study.

S Afr Med J 2013;103(7):467-470. DOI: 10.7196/SAMJ.6636

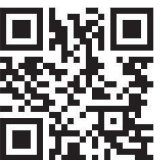

Chronic suppurative otitis media (CSOM) is a chronic infection of the middle ear cleft in which the tympanic membrane is not intact and otorrhoea is present. ${ }^{[1,2]}$ The prevalence of CSOM in sub-Saharan Africa is between $0.4 \%$ and $4.2 \%$, with $>50 \%$ of cases occurring in children under the age of 10 years. ${ }^{[2-5]}$

While the literature reports that the three organisms most commonly cultured in CSOM are Pseudomonas aeruginosa, Staphylococcus aureus and Klebsiella ssp, ${ }^{[6]}$ a previous study at Universitas Academic Hospital (UAH) found the most common bacteria to be Proteus mirabilis (29.0\%), followed by P. aeruginosa (23.7\%). ${ }^{[7]}$ In 2007, the incidence of tuberculosis in South Africa (SA) was estimated at $600-800$ per 100000 population, and since has increased. ${ }^{[8]}$ Studies from the early twentieth century found the prevalence of tuberculous CSOM in patients with CSOM to be between $1 \%$ and $5 \% \cdot{ }^{[9,10]}$ However, a previous study at our hospital found Mycobacterium tuberculosis in only $1 / 114(0.9 \%)$ ears with CSOM. ${ }^{[7]}$

CSOM may result in hearing loss, which can have a considerable negative effect on children's speech, language and cognitive development and on school performance. ${ }^{[11,12]}$

\section{Objectives}

To describe the otological, audiological and bacteriological findings in patients with CSOM and to assess the prevalence of tuberculous CSOM in children seen at the Ear, Nose and Throat (ENT) Clinic at UAH, Bloemfontein, Free State Province.

\section{Methods}

This was a prospective, descriptive study with a cross-sectional analytical component. Ethical approval for the study was obtained from the ethics committee of the Faculty of Health Sciences, University of the Free State.

We included all patients $<13$ years of age with CSOM, with otorrhoea for $>4$ weeks, examined at the ENT clinic at UAH between August 2009 and December 2010. Our exclusion criteria included: if the otorrhoea was due to otitis externa; if there was a stable, dry perforation on otoscopy; if there was otorrhoea with a grommet in situ; or if the parents did not give written consent to participate in the study.

The external ear canal was cleaned using a microscope and handheld low-pressure suction and the tympanic membrane and middle ear mucosa were inspected. Two pus swabs were taken from each affected middle ear: 1 for routine microbiology, culture and fungal cultures and 1 for $M$. tuberculosis culture, taking care to limit contamination from the external ear canal. Details regarding the duration of symptoms, treatment received and response to treatment were recorded. Patients underwent voluntary HIV testing after appropriate counselling by HIV enzyme-linked immunosorbent assay (ELISA) ( $>18$ months of age) or HIV polymerase chain reaction (PCR) ( $<18$ months of age). Children who had previously been diagnosed with HIV were not re-tested. $\mathrm{CD} 4^{+} \mathrm{T}$-cell counts were determined for HIV-positive children according to the age of the child. ${ }^{[13]}$ The height and weight of all patients were plotted on standard World Health Organization (WHO) growth curves. 
Audiological testing was performed once the otorrhoea had resolved. Air conduction thresholds were recorded by play or pure-tone audiometry, as appropriate for the age of the child, in a soundproof booth with a calibrated diagnostic audiometer (Madsen OB822 or Interacoustic $\mathrm{AC} 33$, respectively). When possible, bone conduction thresholds were measured. The pure tone average (PTA) was calculated as the average of the hearing thresholds at $500 \mathrm{~Hz}, 1000$ $\mathrm{Hz}$ and $2000 \mathrm{~Hz}$.

Results are represented by frequencies and percentages. Groups were compared using $t$-tests, chi-squared or Fischer's exact tests, as appropriate.

\section{Results}

Ninety-two children were enrolled in the study, but data collection was incomplete in 6, leaving 86 cases for analysis, comprising 45 males $(52.3 \%)$ and 41 females (47.7\%). The median age was 4.6 years (range 1 - 12 years). Of the 113 ears evaluated, 59 (68.6\%) patients had unilateral disease and 27 (31.4\%) had bilateral disease with a slight right-sided predominance in 59 (52.2\%) ears.

The mean duration of otorrhoea was 161.7 weeks (range 4 - 572 weeks). Referrals came mainly from district hospital doctors (32.6\%) and paediatricians in the public sector (31.4\%), with relatively few referrals from the primary health sector (Table 1). There was a parental complaint of hearing loss in 48 (55.8\%) children. Five (5.8\%) patients had a history of prior adenoidectomy or adenotonsillectomy. Ventilation tubes had been inserted in 7/113 (6.2\%) ears, but no patient had a ventilation tube in situ when entered into the study. No tympanoplasties had been performed but 1 patient had undergone tympanomastoidectomy at another hospital. The parents did not know the indication for this procedure.

In the 3 months prior to enrolment, 55 (48.6\%) ears in 42 (48.8\%) patients had not received any treatment for CSOM while 58 (51.4\%) ears had been treated. Eight (7.1\%) ears were treated with topical acetic acid drops; 18 (15.9\%) with topical quinolones; 26 (23.0\%) with oral antibiotics; 8 (7.1\%) with the combination of acetic acid drops and oral antibiotics and 4 (3.5\%) with the combination of oral and topical antibiotics. Of the 16 ears treated with topical acetic acid drops, 2 (12.5\%) had improved with treatment. Ten (45.4\%) of the ears treated with the topical antibiotics had responded to the initial treatment while $11 / 38(28.9 \%)$ ears treated with oral antibiotics initially responded. We were unable to document the class of antibiotic used since the children's parents did not usually know which treatment was used.

Of the 113 tympanic membrane perforations, 25 (22.1\%) were marginal perforations and $88(77.9 \%)$ were central perforations. Ninety-six (85.0\%) had a thickened appearance, with $63(55.8 \%)$ displaying focal areas of granulation tissue. Three (2.7\%) tympanic membranes had areas of myringosclerosis and 14 (12.4\%) were inflamed. The middle ear mucosa was oedematous in 80 (70.8\%) ears

Table 1. Referring healthcare worker $(N=86)$

\begin{tabular}{ll}
\hline Referral & $\boldsymbol{n}(\%)$ \\
\hline District hospital doctor & $28(32.6)$ \\
Public sector paediatrician & $27(31.4)$ \\
Primary healthcare doctor & $15(17.4)$ \\
Private general practitioner & $11(12.8)$ \\
Private paediatrician & $1(1.2)$ \\
Audiologist & $3(3.5)$ \\
Other & $1(1.2)$
\end{tabular}

and appeared inflamed in 76 (67.3\%). The affected middle ear spaces contained granulation tissue in $42(37.2 \%)$ ears. Cholesteatoma was present in 23 (20.4\%) of the 113 ears. None of the patients had a cleft palate or bifid uvula.

We cultured and identified 153 organisms (Table 2). Gramnegative bacteria were present in $93(82.3 \%)$ ears. $P$. mirabilis was the most common organism in 33 (29.2\%) affected ears, followed by P. aeruginosa in $22(19.5 \%)$ ears. No organisms were cultured in 6 (5.3\%) of the affected ears. Gram-negative bacteria were susceptible to ciprofloxacin in $94.8 \%$ of cases. M. tuberculosis was cultured from 1 HIV-negative child, who also had coalescent mastoiditis and a subperiosteal abscess but did not have facial nerve palsy.

The same bacteria were cultured from both ears in 15/27 (55.5\%) children with bilateral perforations. In $9(33.3 \%)$ patients, the same bacteria were found with 1 additional pathogen cultured in 1 of the ears, while in $3(11.1 \%)$ cases the bacteria differed between the 2 ears.

Thirty-five patients were known to be HIV-positive with 23 (65.7\%) receiving antiretroviral treatment. Forty-eight of the 51

\section{Table 2. Bacteria cultured $(N=113)$}

\begin{tabular}{ll}
\hline Bacteria & $n(\%)$ \\
\hline Gram-negative aerobes & \\
Proteus mirabilis & $33(29.2)$ \\
Pseudomonas aeruginosa & $22(19.5)$ \\
Haemophilus influenzae & $22(19.5)$ \\
Providencia stuartii & $7(6.2)$ \\
Escherichia coli & $6(5.3)$ \\
Klebsiella oxytoca & $4(3.5)$ \\
Klebsiella pneumonia & $4(3.5)$ \\
Citrobacter freundii & $2(1.8)$ \\
Proteus vulgaris & $2(1.8)$ \\
Acinetobacter baumannii & $2(1.8)$ \\
Burkholderia cepacia & $2(1.8)$ \\
Morganella morganii & $2(1.8)$ \\
Enterobacter cloacae & $1(0.9)$ \\
Gram-positive aerobes & \\
Staphylococcus aureus & $7(6.2)$ \\
Streptococcus pyogenes & $3(2.7)$ \\
Enterococcus faecalis & $2(1.8)$ \\
Coagulase neg. Staphylococcus & $2(1.8)$ \\
Streptococcus group G & $1(0.9)$ \\
Streptococcus pneumonia & $1(0.9)$ \\
Streptococcus anginosus & $1(0.9)$ \\
Anaerobes & \\
Bacteriodes thetaiotaomicron & $6(5.3)$ \\
Peptostreptococcus tetradius & $5(4.4)$ \\
Bacteroides ovatus & $4(3.5)$ \\
Fusobacterium necrophorum & $3(2.7)$ \\
Porphyromonas asaccharolytica & $2(1.8)$ \\
Peptostreptococcus anaerobius & $1(0.9)$ \\
Fusobacterium nucleatum & $1(0.9)$ \\
& $1(0.9)$ \\
&
\end{tabular}


patients whose HIV status was not known consented to HIV testing, with 10 (20.8\%) of these patients testing positive. Thus, 45/83 (54.2\%) patients were HIV-positive. $\mathrm{CD} 4^{+} \mathrm{T}$-cell counts were measured in 44/45 HIV-positive children: 22 were immunosuppressed, 4 mildly immunodeficient, 9 moderately immunodeficient and 9 severely immunodeficient. Other co-morbidities included anaemia in 39 (45.3\%) patients and malnutrition - 22/85 (25.9\%) children being underweight and 29/82 (35.4\%) showing stunting.

There was no significant difference in the bacteria cultured between HIV-negative and HIV-positive patients or between the anaemic and non-anaemic patients $(p>0.05)$. Among HIV-positive patients, there was no significant difference between the immunosuppressed and non-immunosuppressed patients. However, P. mirabilis was found significantly more frequently in patients who were underweight (40.9\% v. $17.5 \%$; $p=0.0256)$.

Nine patients (10.5\%) had complications of CSOM with 6 patients having $>1$ complication. Complications occurred in 6/21 (28.6\%) patients (6/23 ears) that had a cholesteatoma (Table 3). Mastoiditis and subperiosteal abscess formation were significantly more common in ears with cholesteatoma than those without $(p<0.0001)$.

Because of difficulty with follow-up and audiology staffing shortages, audiological testing could only be performed in 46 (53.5\%) patients (66 affected ears) (Table 4). Forty-four (66.7\%) of the ears tested had a hearing loss, as defined by a PTA $>25$ decibels hearing level ( $\mathrm{dB} \mathrm{HL})$. Bone conduction thresholds could only be determined for 11 affected ears as a result of the young age of most of the children. Because of the small number, these results are not reported.

There was a parental complaint of hearing loss in 48 (72.7\%) of those ears that underwent audiological testing. The median PTA was $41.7 \mathrm{~dB}$ $\mathrm{HL}$ in ears where there was a complaint of hearing loss compared

Table 3. Ear complications $(N=113)$

\begin{tabular}{lll}
\hline & \multicolumn{2}{c}{$\boldsymbol{n}(\mathbf{\%})$} \\
\cline { 2 - 3 } Complication & $\begin{array}{l}\text { Cholesteatoma } \\
(\mathbf{N = 2 3 )}\end{array}$ & $\begin{array}{l}\text { No cholesteatoma } \\
(\mathbf{N = 9 0 )}\end{array}$ \\
\hline Mastoiditis & $6(26.1)$ & $1(1.1)$ \\
Meningitis & $1(4.3)$ & $2(2.2)$ \\
Subperiosteal abscess & $6(26.1)$ & $0(0)$ \\
Intracranial abscess & $1(4.3)$ & $0(0)$ \\
Facial nerve palsy & $1(4.3)$ & $0(0)$
\end{tabular}

with $25.0 \mathrm{~dB}$ HL in ears where there was no such complaint $(p=0.05)$. There was no association between the type of perforation (central v. marginal) or the presence of cholesteatoma and a PTA $>25 \mathrm{~dB} \mathrm{HL}$ ( $p=0.5068$ and $p=0.4262$, respectively). There was no correlation between the duration of otorrhoea and audiometric thresholds (Spearman correlation coefficient $\leq 0.2$ for all tested frequencies and for the PTA). The audiometric thresholds did not differ significantly between the HIV-positive and HIV-negative patients $(p>0.05)$, nor did they differ significantly between the immunosuppressed and nonimmunosuppressed HIV-positive patients $(p>0.05)$.

\section{Discussion}

The mean duration of otorrhoea in patients included in the study was 161 weeks (>3 years), which may be due to various factors: (i) lack of access to primary health services; (ii) lack of, or delayed, referral from primary healthcare to ENT services; (iii) tolerance of the otorrhoea by parents and healthcare workers who are often unaware of the serious nature of CSOM; and (iv) limited availability of specialist ENT services in the province. Uncomplicated CSOM is painless and not physically debilitating, thus patients and caregivers may feel no urgency to seek prompt treatment, ${ }^{[12]}$ emphasised by the $32 \%$ of children that were referred from paediatric clinics, most likely being examined primarily for other reasons.

There is a lack of consensus regarding the transition from acute otitis media with perforation of the tympanic membrane to CSOM - the WHO considers CSOM to be indicated if otorrhoea is present for $\geq 2$ weeks ${ }^{[2]}$ while others consider $\geq 6$ weeks. ${ }^{[1]}$ For our inclusion criteria, we chose 4 weeks as the duration of otorrhoea to include as many patients with CSOM as possible, but exclude patients with acute otitis media.

There was an almost equal distribution of male and female patients in our study, in contrast to other studies that found a male predominance. ${ }^{[4,5,14-16]}$ The predominance of unilateral disease in children with CSOM has previously been noted. . $^{[3,12,16-19]}$

Most children who had been treated prior to referral did not respond to the treatment given or relapsed, which was the reason for referral. Treatment failure may be due to failure to combine ear toilet with medical treatment, the use of ineffective treatment or the result of re-infection related to poor hygienic conditions. ${ }^{[2,3]}$ Removal of pus and debris improves the quality of the examination and the efficacy of topical agents in eradicating infections in the middle ear and mastoid. ${ }^{[6]}$ Indeed, ear toilet on its own may be as efficacious as ear toilet combined with ototopical or parenteral treatment, ${ }^{[19]}$ although Smith et al..$^{[2]}$ did not find this to be the case. We did not document if ear toilet had previously been performed in our patients.

Table 4. Pure tone audiometry results

\begin{tabular}{|c|c|c|c|c|c|c|}
\hline \multirow[b]{2}{*}{ Threshold (dB HL) } & \multicolumn{6}{|c|}{$n(\%)$} \\
\hline & $\begin{array}{l}250 \mathrm{~Hz} \\
(N=50)\end{array}$ & $\begin{array}{l}500 \mathrm{~Hz} \\
(N=65)\end{array}$ & $\begin{array}{l}1000 \mathrm{~Hz} \\
(N=66)\end{array}$ & $\begin{array}{l}2000 \mathrm{~Hz} \\
(N=65)\end{array}$ & $\begin{array}{l}4000 \mathrm{~Hz} \\
(N=55)\end{array}$ & $\begin{array}{l}\text { Average } \\
500-2000 \mathrm{~Hz} \\
(N=65)\end{array}$ \\
\hline Median & 45 & 45 & 35 & 35 & 35 & 38.3 \\
\hline$\leq 25$ & $13(26)$ & $19(29.2)$ & $21(31.6)$ & $23(35.4)$ & $14(25.5)$ & $21(32.3)$ \\
\hline $26-40$ & $10(20)$ & $13(20)$ & $15(22.7)$ & $21(32.3)$ & $20(36.4)$ & $13(20)$ \\
\hline $41-55$ & $9(18)$ & $12(18.6)$ & $11(16.7)$ & $9(13.9)$ & $6(10.9)$ & $10(15.4)$ \\
\hline $56-70$ & $8(16)$ & $11(16.9)$ & $11(16.7)$ & $5(7.7)$ & $9(16.4)$ & $13(20)$ \\
\hline $71-90$ & $5(10)$ & $5(7.7)$ & $2(3)$ & $0(0)$ & $0(0)$ & $2(3)$ \\
\hline$\geq 90$ & $5(10)$ & $5(7.7)$ & $6(9)$ & $7(10.8)$ & $6(10.9)$ & $6(9.2)$ \\
\hline
\end{tabular}


Only $2 / 16$ ears treated with acetic acid drops improved. This lack of effectiveness of acetic acid has been documented in a recent SA study. ${ }^{[21]}$ CSOM is a condition occurring mainly in patients of low socioeconomic status, ${ }^{[2,12,18]}$ who may not have access to clean water. Exposure of the auditory canals to contaminated water has been identified as a risk factor for CSOM. ${ }^{[19]}$

$P$. mirabilis and $P$. aeruginosa were identified as the most prevalent pathogens, in keeping with previously reported data. ${ }^{[7]}$ There was a low prevalence of $S$. aureus, in contrast to other studies in the literature that found $S$. aureus to be one of the most prevalent bacteria in CSOM. ${ }^{[4,5,14-16,21]}$ An Angolan study in which $64 \%$ of children with CSOM were HIV-positive also found Proteus spp. to be the most commonly cultured bacteria. ${ }^{[18]}$ The authors speculated that this was a result of the high rate of HIV infection predisposing patients to infections by low-virulence bacteria. However, we did not find a significant difference in the bacteria cultured between the HIVpositive and HIV-negative patients, nor was there a significant difference between the HIV-positive patients who were immunosuppressed and those who were not immunosuppressed. Notably, P. mirabilis was cultured significantly more commonly in children who were malnourished. Despite the high prevalence of HIV infection in our patients and the high prevalence of tuberculosis in SA, only 1 case of tuberculous CSOM was detected, in an HIV-negative patient.

In $44.4 \%$ of patients with bilateral disease there was a difference in the bacteriology between the 2 ears, indicating that separate pus swabs need to be taken in cases in which both ears are infected. Despite the fact that quinolones as ear drops have been used to treat CSOM for \pm 15 years, $94.8 \%$ of Gram-negative bacteria identified were sensitive to these agents. The use of ototopical quinolones has not been found to lead to the development of resistant pathogens since the antibiotic concentration achieved is thousands of times higher than the minimum inhibitory concentration required to cause bacterial death. ${ }^{[22]}$ As oral quinolones are not used in public sector clinics to treat children, there is less selective pressure on bacterial populations and thus less resistance to the quinolones in children.

Cholesteatoma was present in $23(20.4 \%)$ ears, with mastoiditis and intracranial complications occurring more often in ears with a cholesteatoma. As cholesteatoma is a disease requiring surgical intervention, delayed referral for CSOM can delay the diagnosis of cholesteatoma and subsequent management, which can lead to a higher incidence of serious and life-threatening complications.

When audiological testing could be performed, there was a tendency towards statistical significance in the worse PTA in ears where there was the symptom of hearing loss compared with ears where there was no parental complaint of hearing loss. A Kenyan study found that $69.7 \%$ of parents of children with hearing loss were aware of the hearing loss, with parental awareness in all cases of children with hearing loss due to CSOM. ${ }^{[23]}$ Thus, parental complaints of suspected hearing loss should always be heeded.

Of the patients tested, 44 (67.7\%) had a hearing loss, which was severe or profound in 21 (31.3\%) patients. In contrast, Adoga et al. ${ }^{[15]}$ in Nigeria found hearing loss to be present in all their patients, which was mild in most cases; while Couzos et al. ${ }^{[2]}$ in a study on Australian Aborigines with CSOM, found that $54 \%$ of their patients $>3$ years of age had an average air conduction hearing threshold $>26 \mathrm{~dB}$, but that the hearing loss was mild or moderate in all cases. In a Nigerian study on CSOM in school children, Olatoke et al. ${ }^{[12]}$ found hearing loss to be present in $65.4 \%$ of patients; $38.5 \%$ of patients had mild hearing loss and $26.9 \%$ moderate hearing loss with the presence of hearing loss being associated with significantly worse academic school performance.

Hearing loss is present in $33-38 \%$ of HIV-infected children and may be conductive or sensorineural. ${ }^{[24,25]}$ Although we did not find a significant difference in the hearing thresholds between the HIVpositive and HIV-negative patients, it is possible that HIV infection created a sensorineural hearing loss that contributed to the more severe hearing loss in our patients.

\section{Conclusions}

There was a long delay between the onset of symptoms and accessing specialist ENT services. During this period the majority of children received no or ineffective treatment for their CSOM. Gram-negative aerobes were the most commonly identified bacteria, with most being sensitive to topical quinolones. Oral antibiotic therapy was usually ineffective. Despite the high prevalence of HIV infection, anaemia and malnutrition, tuberculous otitis media was rare. There was a high prevalence of cholesteatoma, mastoiditis, intracranial complications and hearing loss in the children included in this study. The majority of serious complications occurred in patients with cholesteatoma. Since the treatment for cholesteatoma is surgical, prompt referral and surgical management can lead to a decrease in serious and life-threatening complications.

\section{References}

1. Verhoeff M, van der Veen EL, Rovers MM, Sanders EAM, Schilder AGM. Chronic suppurative otitis media: A review. Int J Pediatr Otorhinolaryngol 2006;70(1):1-12. [http://dx.doi.org/10.1016/j.jijporl.2005.08.021] 2. Acuin J. Chronic Suppurative Otitis Media. Burden of Illness and Management Options. Geneva: World Health Organization, 2004

3. Van Hasselt P, Van Kregten E. Treatment of chronic suppurative otitis media with ofloxacin in hydroxypropyl methylcellulose ear drops: A clinical/bacteriological study in a rural area of Malawi. Int J Pediatr Otorhinolaryngol 2002;63(1):49-56. [http://dx.doi.org/10.1016/S0165-5876(01)00648-6] Nwabuisi C, Ologe FE. Pathogenic agents of chronic suppurative otitis media in Ilorin, Nigeria. East Afr Med J 2002;79(4):202-205. [http://dx.doi.org/10.4314/eamj.v79i4.8879]

. Ahmad B, Kudi M. Chronic suppurative otitis media in Gombe, Nigeria. Niger J Surg Res 2004;5(3):120-123. [http://dx.doi.org/10.4314/njsr.v5i3.12253]

6. Hannley MT, Denneny JC, Holzer SS. Use of ototopical antibiotics in treating 3 common ear diseases. Otolaryngol Head Neck Surg 2000;122(6):934-940. [http://dx.doi.org/10.1067/mhn.2000.107813]

7. Seedat RY, Bester A, Möller E, et al. Bacteriology of chronic suppurative otitis media in the era of Seedat RY, Bester A, Möller E, et al. Bacteriology of chronic suppurative otitis media in the era of
ototopical quinolone use. Paper presented at 42 nd Annual Congress of the South African Society of ototopical quinolone use. Paper presented at 42nd Annual Congress of the South Afric

Otorhinolaryngology-Head and Neck Surgery. Cape Town, South Africa, 8-11 October 2006.
South African Department of Health. Tuberculosis Strategic Plan for South Africa, 2007-2011. 8. South African Department of Health. Tuberculosis Strategic Plan for South Africa, 2007-2011.
Pretoria: Department of Health, 2007. http://www.info.gov.za/view/DownloadFileAction?id=72544 Pretoria: Department of Health, 2007. http://www.info.gov.za/view/DownloadFileAction?id=7254
(accessed 9 September 2012)

9. Jeanes AL, Friedmann I. Tuberculosis of the middle ear. Tubercle 1960;41(2):109-116. [http://dx.doi. org/10.1016/S0041-3879(60)80003-7]

0. M'Cart HWD. Tuberculous disease of the middle ear. J Laryngol Otol 1925;40(7):456-466. [http:// dx.doi.org/10.1017/S0022215100027626]

11. Skarżyński H, Piotrowska A. Screening for pre-school and school-age hearing problems: European Consensus Statement. Int J Pediatr Otorhinolaryngol 2012;76(1):120-121. [http://dx.doi.org/10.1016/j.jporl.2011.10.016]

12. Olatoke F, Ologe FE, Nwawolo CC, Saka MJ. The prevalence of hearing loss among schoolchildren with chronic suppurative otitis media in Nigeria, and its effect on academic performance. Ear Nose Throat J 2008;87(12):E19.

13. World Health Organization. WHO case definitions of HIV for surveillance and revised clinical staging and immunological classification of HIV-related disease in adults and children. Geneva: WHO, 2007.

14. Thorp MA, Gardiner IB, Prescott CA. Burow's solution in the treatment of active mucosal chronic Thorp MA, Gardiner IB, Prescott CA. Burow's solution in the treatment of active mucosal chronic
suppurative otitis media: Determining an effective dilution. J Laryngol Otol 2000;114(6):432-436. suppurative otitis media: Determining an effect
[http://dx.doi.org/10.1258/0022215001906002]

15. Adoga A, Nimkur T, Silas O. Chronic suppurative otitis media: Socio-economic implications in a tertiary hospital in Northern Nigeria. Pan Afr Med J 2010;4(3). [http://dx.doi.org/10.4314/pamj.v4i1.53613]

16. Ferede D, Geyid A, Lulseged S, Melaku A. Drug susceptibility pattern of bacterial isolates from children with chronic suppurative otitis media. Ethiopian J Health Dev 2001;15(2):89-96. [http://dx.doi. org/10.4314/ejhd.v15i2.9882

17. Adhikari P, Joshi S, Baral D, Kharel B. Chronic suppurative otitis media in urban private school children of Nepal. Braz J Otorhinolaryngol 2009;75(5):669-672. [http://dx.doi.org/10.1590/S1808-86942009000500010]

18. Taipale A, Pelkonen T, Taipale M, Bernardino L, Peltola H, Pitkäranta A. Chronic suppurative otitis media in children of Luanda, Angola. Acta Paediatr 2011;100(8):e84-e88. [http://dx.doi.org/10.1111/ j.1651-2227.2011.02192.x]

19. Eason RJ, Harding E, Nicholson R, Nicholson D, Pada J, Gathercole J. Chronic suppurative otitis media in the Solomon Islands: A prospective, microbiological, audiometric and therapeutic survey. NZ Med
ind 1986;99(812):812-815

20. Smith AW, Hatcher J, Mackenzie IJ, et al. Randomised controlled trial of treatment of chronic suppurative otitis media in Kenyan schoolchildren. Lancet 1996;348(9035):1128-1133. [http://dx.doi. org/10.1016/S0140-6736(96)09388-9]

21. Loock JW. A randomised controlled trial of active chronic otitis media comparing courses of eardrops versus one-off topical treatments suitable for primary, secondary and tertiary healthcare settings. Clin Otolaryngol 2012;37(4):261-270. [http://dx.doi.org/10.1111/j.1749-4486.2012.02532.x]

22. Couzos S, Lea T, Mueller R, Murray R, Culbong M. Effectiveness of ototopical antibiotics for chronic suppurative otitis media in Aboriginal children: A community-based, multicentre, double-blind randomised controlled trial. Med J Aust 2003;179(4):185-190.

23. Omondi D, Ogol C, Otieno S, Macharia I. Parental awareness of hearing impairment in their school-going children and healthcare seeking behaviour in Kisumu district, Kenya. Int J Pediatr Otorhinolaryngol 2007;71(3):415-423. [http://dx.doi.org/10.1016/jijporl.2006.11.007]

24. Christopher N, Edward T, Sabrina B-K, Agnes N. The prevalence of hearing impairment in the 6 months 5 years HIV/AIDS-positive patients attending paediatric infectious disease clinic at Mulago Hospital. Int J
J 5 years HIV/AIDS-positive patients attending paediatric infectious disease clinic at Mulago $\mathrm{H}$
Pediatr Otorhinolaryngol 2013;77(2):262-265. [http://dx.doi.org/10.1016/jijporl.2012.11.013]

25. Chao CK, CzechowiczJA, Messner AH, et al. High prevalence of hearing impairment in HIV-infected Peruvian children. Otolaryngol Head Neck Surg 2012;146(2):259-265. [http://dx.doi.org/10.1177/0194599811429271]

Accepted 22 April 2013 